EDITORIAL

\title{
The Need for Surveillance of Antimicrobial Resistance in Developing Countries: Practice Makes Perfect \\ Ahmed Morad Asaad
}

Medical Microbiology and Immunology Department, Faculty of Medicine, Zagazig University, Zagazig, Egypt

Corresponding Author:

Ahmed Morad Asaad

Medical Microbiology and Immunology Department, Faculty of Medicine, Zagazig University, Zagazig, Egypt

E-mail: ahmedmoradasaad@ hotmail.com

$\mathrm{F}$ or several decades, antimicrobial agents have been regarded as the magic key to cure infections. However, use of antibiotics has become widespread, and these drugs have been extensively misused in both humans and food-producing animals in ways that favour the selection and spread of resistant bacteria. The scenario is more dramatic as the pipeline for the development of new antibacterial drugs is now virtually empty ${ }^{[1]}$.

According to WHO reports, it is estimated that 300 million people will die prematurely because of infections as a result of multidrugresistant (MDR) organisms over the next 35 years. Besides, the world can expect to lose 60-100 trillion Dollars in economic output if antimicrobial drug resistance is not effectively tackled $^{[2,3]}$

Several strategies have been designed to control the spread of antimicrobial resistance (AMR) including: use of antibiotic policies or full stewardship programs, innovative vaccine technologies, use of monoclonal antibodies, gut microbiome interventions and phage therapy. Yet, no single or simple strategy will suffice to fully contain the emergence and spread of MDR organisms ${ }^{[4]}$.

The collection of reliable information about the antimicrobial resistance situation inside hospitals through well-conducted surveillance system is essential to inform strategies and prioritize interventions to tackle the problem. Surveillance activities could provide the necessary information to develop and monitor therapy guidelines, antibiotic formularies, antibiotic stewardship programs, public health interventions, infection control policies, and novel antimicrobials and vaccines. WHO has for many years promoted the global monitoring of AMR and taken steps to raise awareness of the impending public health crisis it will cause ${ }^{[1,5]}$.

Although AMR surveillance has been undertaken for many years in a number of high-income countries, there are still large gaps in knowledge about the status of AMR surveillance capacities in developing countries with resource-limited settings ${ }^{[6]}$.

Since we are in the era of computers and software programs, only information technology systems can readily manage and consolidate data to allow timely and accurate analysis locally, nationally and at a global level. Several open-access software programs are available for clinical application in healthcare settings including SatScan, FlexScan, WHONET, HealthMap and FluNet. The WHONET; a desktop based software, is recommended by WHO for clinical microbiologists and infection control practitioners. This program could manage laboratory microbiological data and antimicrobial susceptibility testing results. The program implements a data input interface, validation rules, alerts and storage. This free program has been used in over 3000 hospitals from 200 countries, worldwide ${ }^{[5,7]}$. As of September, 2017, the WHO Regional Office has successfully conducted a workshop 
in Cairo gathering 16 experts from 8 countries in the Eastern Mediterranean Region. The main objective of this workshop was to introduce WHONET software as a paramount tool for enhancing the local use of surveillance data in healthcare settings, and create a provisional pool of WHONET super users for the Region who can provide technical support to clinical microbiologists and infection control practitioners in the Eastern Mediterranean Region ${ }^{[8]}$.

In conclusion, it is the time for all hospitals in Egypt to implement surveillance activities and enhance monitoring of AMR. The need for open-access, generic and user friendlyinformation technology as WHONET program seems crucial for better understanding of the local epidemiology of microbial populations, more-definitive selection of empiric antibiotic policies and precise identification of hospital or community outbreaks.

\section{Declaration of interest}

The authors report no conflicts of interest. The authors alone are responsible for the content and writing of the paper.

\section{Funding information}

None declared

\section{REFERENCES}

1- Tilotson GS, Zinner SH. Burden of antimicrobial resistance in an era of decreasing susceptibility. Exper Rev Anti Infect Ther 2017, 15 (7): 663-676.

2- Alsan M, Schoemaker L, Eggleston K, Kolli P, Bhattacharya J. Out-of-pocket health expenditures and antimicrobial resistance in low-income and middle-income countries; an economic analysis. Lancet Infect Dis 2015; 15 (10): 1203-1210.

3- Klein EY, Van Boeckel TP, Martinez EM, Pant S, Gandra S, Levin SA et al. Global increase and geographic convergence in antibiotic consumption between 2000 and 2015. Proc Natl Acad Sci USA. 2018;115(15):E3463-70.

4- Mantravadi PK, Kalesh KA, Dobson RC, Hudson AO, Parthasarathy A. The quest for novel antimicrobial compounds: Emerging trends in research, development, and technologies. Antibiotics 2019, 8(1): 8. https://doi.org/10.3390/antibiotics8010008

5- WHO. Antibiotic resistance. Geneva: World Health Organization; 2018.

6- Van Dijck C, Vliegheb E, Cox JA. Antibiotic stewardship interventions in hospitals in lowand middle-income countries: a systematic review. Bull World Health Organ 2018; 96: $266-280$.

7- Natale A, Stelling J, Meledandri M, Messenger LA, D'Ancona F. Use of WHONET-SaTScan system for simulated real-time detection of antimicrobial resistance clusters in a hospital in Italy, 2012 to 2014. Euro Surveill 2017, 22 (11): 30484.

8- WHO. Training of WHONET super users in the Eastern Mediterranean Region. Geneva. World Health Organization; 2017. 